\title{
"Oh yes, oh yes, these are the atoms!" A Personal Recollection from the Times of the Invention of the STM
}

\author{
Karl-Heinz Rieder*
}

\begin{abstract}
The scanning tunneling microscope, invented and developed in the 1980s in the IBM research laboratory in Rüschlikon, has become the dominent scientific tool in surface science and together with its younger brother the atomic force microscope, is widely used also in many other modern research areas. This account contains very personal anecdotal memories from a colleague who worked in a neighboring lab in Rüschlikon describing some events at the periphery of the development of this ingenious instrument.
\end{abstract}

Keywords: Physics Nobel Prize 1986 · STM invention · Surface science

From 1975 to 1986 I was research staff member at IBM Research Laboratories in Rüschlikon near Zürich. There I got to know Gerd Binnig as he joined the group of Heini Rohrer, a renowned expert for phase transitions and critical phenomena in magnetic materials. Heini and Gerd wanted to move into a new area of research, namely, vacuum electron tunneling. Their original intention was a new spectroscopic method with a high lateral resolution. Gerd Binnig showed his talent not only in the laboratory, but also in our IBM music band. We put a lot of effort into practicing and performing some of his songs (Fig. 1).

After having measured successfully the exponential current-voltage characteristics for tunneling in vacuum, which meant that they had already solved serious mechanical damping problems, Binnig and Rohrer started to scan the counter electrode, the

\footnotetext{
${ }^{\star}$ Correspondence: Prof. Dr. K.-H. Rieder Nanoscale Materials Science Empa, Swiss Federal Laboratories for Materials Science and Technology

Überlandstrasse 129

$\mathrm{CH}-8600$ Dübendorf

Tel.: + 41587654629

Fax: + 41587654034

E-mail: karl-heinz.rieder@empa.ch
}

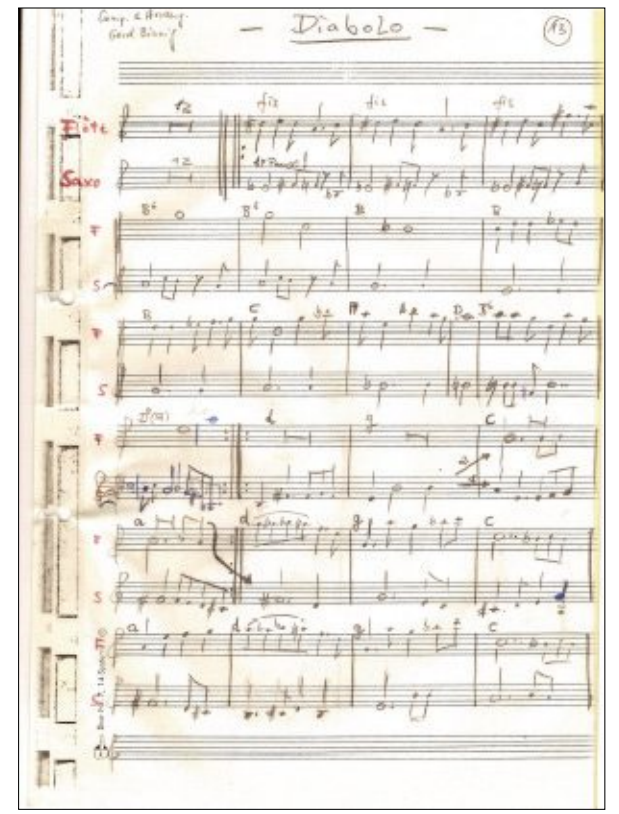

Fig. 1. Gerd Binnig's composition named 'Diabolo'.

tunneling tip, over surfaces. Surprisingly they quickly were able to 'see' atomic steps with their first STM, and already these results attracted considerable interest. After showing these step corrugations to me, it immediately came to my mind that they were now in a position to look at some longstanding problems in surface science with their new instrument. The structure of surfaces has intrigued me for a long time, and I used helium atom diffraction in order to study clean surfaces or adsorbate structures. ${ }^{[1]}$ So I suggested to Binnig and Rohrer to study reconstructed gold and silicon surfaces, which were known to deviate from the bulk-derived structures.
These surfaces had been investigated with various methods without conclusive results. Together with these problems I also provided my well-prepared single crystals. Because of its relative large corrugation, I proposed to try the $(1 \times 2)$-reconstructed gold(110) surface first. Previous helium scattering experiments suggested either a symmetric missing-row or an asymmetric saw-tooth reconstruction. Before returning that crystal to me in form of a solidified droplet, Binnig had succeeded in imaging the $(1 \times 2)-A u(110)$ structure.

I recall that I returned from a conference late one evening and found the corresponding STM line scans on my desk (Fig. 2). In order to document his success, Binnig had left this 'STM image' there for me. I realized instantly from the symmetric shape of line scans, that gold(110) is missing-row reconstructed. I remember vividly that I kept looking at this image literally for hours, desperately trying to find any reason for which helium scattering might be

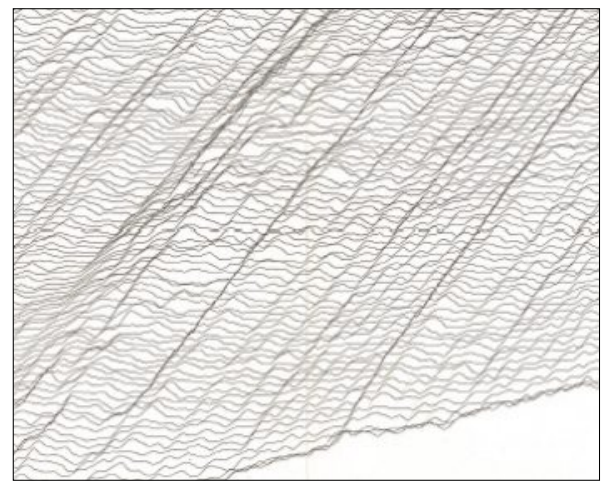

Fig. 2. The first STM image of the $(1 \times 2)$ missing-row reconstructed $\mathrm{Au}(110)$ surface. 
good for in the future. I came to the conclusion that Binnig and Rohrer's new method would make helium scattering only valuable for studying structures of insulating surfaces. But Binnig, Gerber and Quate's invention of the atomic force microscope five years later made my favorite investigation technique basically obsolete.

Binnig went on to investigate the complex reconstruction of $\mathrm{Au}(100)$ and showed that single atoms could be resolved with STM - before melting this crystal as well.

Numerous scientists from all over the world now came to visit our laboratory, but often expressed doubts about these novel STM results. The inventors, how ever, were absolutely confident and I heard many times Christoph Gerber, a member of Binnig and Rohrer's team and co-inventor of the STM, explaining results to visitors and emphasizing "Oh yes, oh yes, we see the atoms!"

The final breakthrough, convincing the entire scientific community, came by solving the structure of the reconstructed $(7 \times 7)$ silicon(111) surface. This established STM as a powerful surface science tool and added another melted crystal to my collection.

Soon it became clear that the invention of the STM had a good chance to be awarded the Nobel Prize. On October $15^{\text {th }} 1986$ I came to the laboratory in the early afternoon and found the whole
IBM Rüschlikon in great excitement, because Binnig and Rohrer had received the phone call from Stockholm. I immediately walked up to them to congratulate the new laureates. Binnig's comment to me was: "But I'll get only one quarter!" Completely stunned with this reply, I told him that in the future his name would stand in a row together with such famous physicists as Einstein, Planck, Dirac and Feynman. But Gerd replied dryly: "It's nevertheless only one quarter!"

Received: December 11, 2011

[1] K.-H. Rieder, T. Engel, Phys. Rev. Lett. 1980, 45,824 . 